\title{
Ostdeutsche Identität(en) im Wandel? Perspektiven für Intra- und Interkohortenvergleiche
}

\author{
Lars Vogel · Julia Leser
}

Eingegangen: 6. November 2019 / Überarbeitet: 17. Juni 2020 / Angenommen: 22. September 2020 /

Online publiziert: 20. Oktober 2020

(C) Der/die Autor(en) 2020

Zusammenfassung Der Beitrag analysiert ostdeutsche Identitätsbildungsprozesse bei jungen Erwachsenen im Jahr 2004 und rund zehn Jahre später. Empirische Basis ist ein Intra- und Interkohortenvergleich der Befunde von drei qualitativen Studien basierend auf Leitfadeninterviews und Gruppendiskussionen. Dieser diachrone Vergleich zeigt partielle Kontinuitäten in den Identitätskonstruktionen der ostdeutschen Befragten: Die wahrgenommene Abwertung Ostdeutschlands bildet weiterhin den zentralen Ausgangspunkt der Identifizierung als ostdeutsch und die Reaktionen darauf sind ebenso weiterhin heterogen. Diese Heterogenität wird zu einer Typologie verdichtet: Manche Ostdeutsche zielen auf eine Aufwertung ihrer kollektiven Identität durch Umdeutung und beziehen sich dabei auf kollektivistische Werte oder die gelungene Bewältigung der Systemtransformation. Andere lehnen die Identifikation als ostdeutsch und die Klassifizierung in Ost und West als überholt und illegitim ab. Allerdings lässt der nach 1990 geborene Teil dieser Gruppe zusätzlich individuelle Strategien instrumenteller Identitätsbildung erkennen. Diese Heterogenität ostdeutscher Identität(en) kann auf Kohortenunterschiede und soziale Merkmale der Probanden zurückgeführt werden. Zugleich wird der Einfluss des Forschungsdesigns und insbesondere der Fallauswahl der einbezogenen Studien auf die Ergebnisse methodologisch diskutiert. Die Befunde dienen damit als Ausgangspunkte für zukünftige Intra- und Interkohortenvergleiche, in denen die individuellen und methodischen Einflussfaktoren für Identitätsbildungsprozesse stärker berücksichtigt werden sollten.

\footnotetext{
Dr. L. Vogel $(\bowtie)$

Arbeitsbereich Empirische Methoden und politische Soziologie, Institut für Politikwissenschaft, Universität Leipzig, Beethovenstraße 15, 04107 Leipzig, Deutschland

E-Mail: lars.vogel@uni-leipzig.de

Dr. J. Leser

BMBF-Forschungsprojekt „Fremde im eigenen Land? Eine Studie über die Veränderbarkeit nationaler Narrative mithilfe Politischer Laboratorien (PoliLab)“, Institut für Politikwissenschaft, Universität Leipzig, Beethovenstraße 15, 04107 Leipzig, Deutschland
} 
Schlüsselwörter Ostdeutschland · Ostdeutsche Identität · Kollektive Identität · Klassifizierung $\cdot$ Kohortenstudien

\title{
East German identities in flux? Avenues for intra- and inter-cohort comparisons
}

\begin{abstract}
This paper analyses the self-identification of young adults as Eastern Germans. It is based on intra- and inter-cohort comparisons of guided interviews and group discussions conducted in 2004 and around ten years later. The results provide empirical evidence of partial continuities in the identification process of young Eastern Germans. In particular, the perceived devaluation of Eastern Germany remains a major starting point for self-identification as Eastern German. Furthermore, the response strategies to curb the devaluation continue to be heterogeneous. From this the paper develops a typology, namely that some Eastern Germans claim a positive interpretation of their Eastern German identity either based on collectivism or on their successful adaptation following the transformation after 1989. Others reject self-identification as Eastern German and reject the East-West categorisation completely. Additionally, some of those born after 1990 respond individually and instrumentally to their classification as Eastern German. This heterogeneity is due to both cohort differences and the social characteristics of the interviewees. Accordingly, the methodological section of this paper discusses the impact of the research design, with particular focus on the sample selection of the included studies on their outcomes. The results serve as starting point for further intra- and inter-cohort comparisons, which should pay more attention to the individual and methodological conditions for political self-identification.
\end{abstract}

Keywords Eastern Germany · East German identity · Collective identity · Classification $\cdot$ Cohort studies

\section{Die Diskussion um ostdeutsche Identität(en)}

In der öffentlichen Diskussion über kollektive Identitäten - seien sie regional, national oder europäisch - spielt Ostdeutschland in mehrfacher Hinsicht eine prominente Rolle. Umfrageergebnisse (z. B. Reiser et al. 2018) legen nahe, dass in Ostdeutschland solche Nationalstaatskonzeptionen stärkere Unterstützung finden, die ethnische oder kulturelle Homogenität anstreben. Ferner wird die höhere Unterstützung fremdenfeindlicher Positionen - oder auch die in Ostdeutschland weiter verbreitete Unzufriedenheit mit der Demokratie - häufig in Verbindung mit einer ostdeutschen Identität gebracht, die auf der Basis der ostspezifischen Geschichte, d. h. den Erfahrungen in der DDR-Diktatur und den Abwertungserfahrungen der Transformationszeit, entstanden sei und potentiell in einem Spannungsverhältnis zu einer gesamtdeutschen oder europäischen Identität stehe (z. B. Best et al. 2014; Quent 2012). Im Kontrast zu diesen Deutungsversuchen ostdeutscher Identität stehen solche, in denen die erfolgreiche Bewältigung der Transformation zur Basis der ostdeutschen Identität gemacht wird. Damit gingen Erfahrungen und Kompetenzen einher, die als Berei- 
cherung gesamtdeutscher Diskussions- und Entscheidungsprozesse gedeutet werden (z. B. Lettrari et al. 2016).

Bereits seit Mitte der 1990er Jahre wurde untersucht, ob es eine ostdeutsche kollektive Identität gibt, auf welcher Basis sie entsteht und welche Folgen sie zeitigt. Zunächst lag der der Fokus in der politischen Kulturforschung, die nach Erklärungen für konstatierte Ost-West-Unterschiede in den politischen Einstellungen suchte. Zwei Erklärungsansätze wurden entwickelt: Nach der Sozialisationshypothese sei die ostdeutsche Identität eine Folge der Sozialisation in der DDR, nach der Situationshypothese sei sie hingegen eine Reaktion auf gegenwärtige Problemlagen und eine Symbolisierung der sozialen Position der Ostdeutschen im Vergleich zu den Westdeutschen (Kaase und Bauer-Kaase 1998; Pickel und Pollack 1998). Die ostdeutsche Identität wurde auch später als Reaktion auf die Abwertung ostdeutscher Biografien und Erfahrungen und die relative kollektive Deprivation Ostdeutscher gegenüber den Westdeutschen verstanden. Diese „Abwertungsmechanismen“ (Kollmorgen 2011, S. 302) konstruierten und reproduzierten Ostdeutschland als soziale Kategorie, die von der westdeutschen Normalität abweiche (Roth 2008). Die Ostdeutschen werden damit zur symbolisch ausgeschlossenen (Kubiak 2018) oder gar „,verlorenen“ (Kollmorgen und Hans 2011) „Minderheit im eigenen Land“ (den Hertog 2004), die in die gesamtdeutsche Gesellschaft zu integrieren sei.

Auf Basis narrativer Interviews kommt Jaqueline Flack zu dem Schluss, dass sich Vertreter/-innen der Kohorte der zwischen 1975 und 1985 in Ostdeutschland geborenen Personen zwar zunehmend von diesem auch medial dominanten Bild der Ostdeutschen distanzierten, dieses Konstrukt jedoch ,als negative Kontrastfolie im Kontext individueller Identitätsentwürfe erhalten“ bleibe (Flack 2016, S. 67). Die Befragten seien sich über die diskursiven Herstellungsweisen und Zirkulationen der negativen Bilder der Ostdeutschen bewusst und versuchten, diese mit eigenen Identitätsentwürfen zu kontrastieren (ebd.). Als ein prominentes Beispiel aus der öffentlichen Diskussion dazu ist der im Jahr 2012 erschienene Essayband Dritte Generation Ost: Wer wir sind, was wir wollen anzusehen (Hacker et al. 2012). Zudem werden in der jüngeren Diskussion über Ostdeutschland vermehrt Parallelen zur Debatte über Migration und Integration gezogen. Naika Foroutan et al. (2019) sprechen von ,ostmigrantischen Analogien“: Sowohl Migrant/-innen als auch Ostdeutsche würden Erfahrungen der Abstiegsangst, der sozialen Ungleichheit und der politischen Entfremdung teilen und seien ,,von sozialer, kultureller und identifikativer Abwertung betroffen“ (Foroutan et al. 2019, S. 4). Rebecca Pates hat gezeigt, wie „der Ossi““ seit der Wende als Diskursfigur mit ,ungewollte[r] Migrationserfahrung“ produziert wurde (Pates 2013, S. 11; vgl. Ahbe 2004). Daniel Kubiak (2018) zeigte u.a. in dieser Zeitschrift auf der Basis von Imitation-Games und Gruppeninterviews, dass zwischen 1990 und 1995 in Ost- und Westdeutschland Geborene die Klassifizierung der Ostdeutschen als „Othering“ wahrnehmen, diese Zuschreibungen weitertragen und selbst reproduzieren. Die ,wahrgenommene Abwertung [... kann, L.V./J.L.] zu einem verstärkten Zugehörigkeitsgefühl zum Ostdeutschsein“ führen (ebd., S. 35). Diese Prozesse seien auch im Umgang mit Menschen mit Migrationshintergrund zu beobachten (ebd., S. 27).

Vor diesem Hintergrund greift der vorliegende Beitrag die beiden jüngsten Studien zu ostdeutscher Identität auf (Kubiak 2018; Flack 2016) und vergleicht die darin 
beschriebenen Identitätskonstruktionen mit Leitfadeninterviews, die rund zehn Jahre zuvor im Jahr 2004 mit in Ostdeutschland geborenen jungen Erwachsenen geführt und ausgewertet wurden. ${ }^{1}$ Aus methodischer Sicht ist damit eine Kombination aus Inter- und Intrakohortenvergleich angelegt. Die im Jahr 2004 identifizierte Typologie ermöglicht einen diachronen Vergleich, um Kontinuitäten und Verschiebungen in den ostdeutschen Identitätskonstruktionen zu bestimmen. Im Ergebnis zeigt sich eine partielle Kontinuität. Die wahrgenommene Abwertung Ostdeutschlands bildet auch gegenwärtig weiterhin für einen Teil der Probanden den Ausgangspunkt ihrer Identifizierung als ostdeutsch. Dennoch ist es nicht allein diese Wahrnehmung von Abwertung, die eine ostdeutsche Identität entstehen lässt. Insbesondere das Selbstverständnis, durch die Erfahrung der Wiedervereinigung und ihre erfolgreiche Bewältigung besondere Kompetenzen zu besitzen, bildet eine eigenständige Quelle der Identifizierung. Weiterhin lässt sich die 2004 identifizierte Heterogenität der ostdeutschen Identitäten im Vergleich zwischen den beiden jüngeren Studien finden und kann mit Kohortenunterschieden zwischen den vor und nach 1990 Geborenen begründet werden. In den einbezogenen Studien kommen teilweise variierende Methoden der Datenerhebung und der Fallauswahl zum Einsatz. Diese Pluralität bekräftigt die Validität der gefundenen Kontinuität, zugleich werden aber auch die methodischen Unterschiede als mögliche Ursache für inhaltliche Verschiebungen diskutiert. Im Ergebnis eröffnen sich Vergleichsperspektiven, die als Ausgangspunkt für die Weiterentwicklung zu integrierten Kohortenstudien dienen können.

Im folgenden Abschnitt stellen wir zunächst unsere theoretischen Annahmen für die empirische Untersuchung ostdeutscher Identifizierungsprozesse vor. Im Fokus steht die Wechselwirkung zwischen Klassifizierung und individueller Identifizierung, die stets in einen performativen Diskurs eingebettet ist, in der die kollektive Identität behauptet, kritisiert oder verteidigt wird (Delitz 2018, S. 18) und die Rückwirkungen bzw. „looping effects“ (Hacking 1999) auf die individuellen Identifizierungsprozesse besitzt (Pates 2013, S. 12f.). Im Anschluss folgt die Interpretation der 2004 erhobenen Leitfadeninterviews mit ostdeutschen jungen Erwachsenen, aus der eine Typologie ostdeutscher Identifizierungen entwickelt wird. Im abschließenden Teil werden Möglichkeiten für Intra- und Interkohortenvergleiche skizziert, die sich durch die Kontrastierung dieser Typologie mit den Ergebnissen der beiden jüngsten Studien von Flack (2016) und Kubiak (2018) eröffnen.

\section{Die Konstruktion kollektiver Identität zwischen Klassifizierung und Identifizierung}

Identität steht dafür, die Gleichheit von Wahrnehmungen (Kontinuität und Kohärenz) gegenüber deren zugleich bestehender Differenz zu betonen (vgl. Wagner 1999, S. 65). Während individuelle Identität die Kontinuität in der eigenen Biografie und

\footnotetext{
1 Eine weitere Studie (Rippl et al. 2018) analysiert zwar ebenso auf der Basis von Leitfadeninterviews die Selbstidentifikation der Ostdeutschen. Allerdings kann diese Studie nicht in den Vergleich einbezogen werden, weil die Altersspanne der Befragten größer als bei den drei übrigen Studien ist, sodass weder ein Inter- noch ein Intrakohortenvergleich (s. unten) möglich ist.
} 
die Differenz zu anderen Personen betont, liegt der Fokus bei kollektiver Identität auf der Gleichheit mit anderen Personen. Wird in der Selbst- oder Fremddefinition die Gleichheit mit anderen Personen betont, ohne dass daraus Folgen erwachsen, liegt eine Klassifizierung bzw. soziale Kategorie vor. Wenn hingegen für eine Gruppe von Personen die Vorstellung ihrer Gleichheit Folgen für das individuelle Handeln zeitigt, liegt eine kollektive Identität bzw. eine soziale Gruppe vor (Jenkins 1996, S. $81 \mathrm{ff}$.). Somit gibt es keine Gruppenidentität ohne Klassifizierung, aber nicht jede Klassifizierung geht mit einer Identifizierung einher. Die Betonung von Gleichheit unter Vernachlässigung von Differenzen verdeutlicht den konstruktivistischen Charakter kollektiver Identität (Delitz 2018, S. 27). Die Analyse kollektiver Identität untersucht folglich die Prozesse, die zur Konstruktion von Kollektiven und der Identifizierung mit ihnen führen und zwar in ihrer Funktion zur Bildung von Sinnzusammenhängen, die durch die Erzeugung eines Kollektivs ermöglicht werden. Die ,,konstruierten Kategorien oder Identitäten [...formen, L.V./J.L.] Personen, und Personen formen sich gemäß dieser Vorgaben [..., wobei L.V./J.L.] die intersubjektiven Anerkennungs- und asymmetrischen Missachtungsverhältnisse“ zu beachten seien (Emcke 2018, S. 208 ff.). Kollektive Identität wird in einer kontinuierlichen und dynamischen Synthese zwischen Selbst- und Fremddefinitionen gebildet. Diese Synthese- und Aushandlungsleistungen sind inhärent konflikthaft (Jenkins 1996, S. $20 \mathrm{ff}$.), da sie auf einer Makroebene, inmitten von Auseinandersetzungen zwischen unterschiedlichen sozialen Gruppen stehen, die bemüht sind, eine gegebene Definition eines Identifikationsobjektes zu bewahren oder zu verändern“ (Fach et al. 1998, S. 5 f.). Auf der Mikroebene reagieren die individuellen Akteure auf die Klassifizierungen und Identifizierungsangebote in Abhängigkeit ihrer Interessen und den ihnen zur Verfügung stehenden Ressourcen (Jenkins 1996, S. 97). Diese „looping effects“ (Hacking 1999) sind vielfältig: Klassifizierungen können wahrgenommen, ignoriert, akzeptiert oder zurückgewiesen werden.

Entscheidend für die Reaktion ist, dass Personen ihre individuelle und kollektive Identität als Mitglied einer Gruppe permanent mit Anderen und mit anderen Gruppen vergleichen (Tajfel und Turner 2004). Dieser Vergleich diene der Bewertung der eigenen Identität in Bezug auf als relevant wahrgenommene Vergleichsdimensionen (Mummendey und Kessler 2000, S. 280). Die Reaktionen auf Fremddefinitionen unterscheiden sich folglich danach, ob der Vergleich auf diesen Dimensionen positiv oder negativ ausfällt und können auf eine Aufwertung der kollektiven Identität, auf eine $\mathrm{Ab}$ - oder Umbewertung der relevanten Vergleichsdimension oder auf individuelle De- oder auch Reklassifizierung zielen. ${ }^{2}$ Die gewählte Strategie hängt u. a. von der wahrgenommenen Durchlässigkeit der Gruppengrenze ab. Darunter wird das Ausmaß verstanden, die selbstdefinierte Zugehörigkeit zu einer Gruppe oder sozialen Kategorie innerhalb einer Interaktion aufrechtzuerhalten (Jenkins 1996, S. 94). Je durchlässiger die Gruppengrenze wahrgenommen wird, umso eher ist eine individuelle Strategie zu vermuten, je undurchlässiger, desto eher eine kollektive Strategie (vgl. Haeger 1998, S. 30). Ein als legitim befundener Unterschied zwischen den

\footnotetext{
2 Der Begriff der Deklassifizierung bedeutet, die Klassifizierung, z. B. wegen eines gemeinsamen Zieles, als nicht relevant abzulehnen. Die Reklassifizierung zielt auf die Aufhebung der Klassifizierung durch die Betonung einer alternativen und relevanteren Kategorie, z. B. als Deutsche (vgl. Haeger 1998, S. 28).
} 
Gruppen lässt ebenso eher die individuelle Strategie vermuten; ein als illegitim befundener die kollektive Strategie (vgl. Mummendey und Kessler 2000, S. 286).

\section{Datenbasis und Methode}

Vor diesem Hintergrund können Interviews als Protokolle einer Identitätskonstruktion verstanden werden, die einen ,Einigungsprozesses von Interviewer und Interviewten auf eine sozial akzeptierte [Identitätsdarstellung, L.V./J.L.]“ (Scherr 1995, S. 40) abbilden können, wobei alle Teilnehmenden auf kollektive Identitätsangebote zurückgreifen und mit unterschiedlichen Ressourcen operieren. Deren Analyse kann die Bedingungen zeigen, unter denen ostdeutsche Identitäten erzeugt oder abgelehnt werden. Hierfür eignen sich insbesondere halbstrukturierte Leitfadenbefragungen, da sie es den Interviewern ermöglichen, den Schwerpunkt der Narration auf kollektive Identitäten zu setzen und es den Interviewten dennoch möglich ist, ihre Selbstdefinition und ihre Reaktion auf die Fremddefinition innerhalb des für sie relevanten Kategoriensystems vorzunehmen. Insbesondere, wenn ostdeutsche Identität nicht aktiv oder lediglich rudimentär thematisiert wird, ist ein Hinweis auf ihre geringe Salienz bei den Befragten gegeben. Halbstrukturierte Befragungen liegen einer Studie von 2004 und den Arbeiten von Kubiak (2018) und Flack (2016) zugrunde. Der Vergleich der drei Befragungen ermöglicht die Exploration der (Dis-)Kontinuität salienter kollektiver Identitätsangebote und der individuellen Reaktionsstrategien darauf.

Die Befragung von 2004 wurde im Rahmen des von Thorsten Oppelland und Andreas Hallermann geleiteten politikwissenschaftlichen Lehrforschungsseminars „Politische Identität: Thüringisch, ostdeutsch, deutsch, europäisch - oder alles zusammen?“" an der Universität Jena durchgeführt. Der Erstautor war als Student an der Erhebung und der Analyse der Interviews im Hinblick auf ostdeutsche Identitäten beteiligt. Es wurden derzeit 37 Einzelinterviews zum Thema kollektive Identität mit jungen Erwachsenen geführt. Nach einer offenen Einleitung zum Thema Identität und Zugehörigkeit thematisierte der Leitfaden die Identifizierung mit den politischen Kollektiven eigene Region, eigenes Bundesland, Ostdeutschland, Deutschland und Europa, wenn die Interviewten diese Gruppenzugehörigkeiten nicht bereits selbst aufgegriffen hatten. Ziel war es, die vermutete Vielfalt kollektiver Identitätsbildungsprozesse explorativ zu kartieren. Die Studierenden erhielten im Rahmen des Seminars eine Einführung in die Durchführung narrativer Interviews. Die Interviewten wurden anschließend durch die Studierenden des Lehrforschungsseminars ausgewählt und an mehreren Orten, vorrangig in Thüringen, interviewt. Durch eine Quotensteuerung gelang eine nahezu paritätische Verteilung von Männern und Frauen und von (gebürtigen) Ost- und Westdeutschen. Die regionale Zuordnung erfolgte allein über den Geburtsort in der DDR bzw. BRD. Parität wurde auch bezüglich der Bildungswege angestrebt, wobei nur zwischen (ehemaligen) Studierenden und Nicht-Studierenden unterschieden wurde, wobei letztere 15 von 37 Interviews ausmachen. Die Interviews wurden transkribiert und in Anlehnung an die qualitative Inhaltsanalyse nach Mayring (2015) paraphrasiert, strukturiert und induktiv zu Typen verdichtet. Die hier dargestellte Analyse konzentriert sich auf das Thema der 
ostdeutschen Identität und analysiert ausschließlich die Interviews mit Personen, die in der DDR zwischen 1975 und 1984 geboren wurden.

Die Erhebungsmethode unterscheidet sich damit nicht von der Studie von Flack (2016), in der ebenfalls Einzelinterviews zur Anwendung kamen. Diese werden zwar als ,narrative Interviews“ (ebd., S. 59) bezeichnet, die publizierten Interviewauszüge zeigen jedoch, dass (Nach-)Fragen nach Art eines Leitfadens das Interview strukturierten. Der Beitrag enthält keine Dokumentation über den thematischen Kontext der durchgeführten Interviews, die Anzahl der Interviews, die Auswahl der Interviewten oder die Komposition des Samples - mit Ausnahme des Geburtsjahres. Der thematische Kontext lässt sich jedoch rekonstruieren, wenn berücksichtigt wird, dass der Beitrag in dem Sammelband Die Generation der Wendekinder (Lettrari et al. 2016) erschien. Dieser Fokus prägt die Auswahl der Probanden, die allesamt zwischen 1975 und 1985 in der DDR geboren wurden. Dass diese Generation durch die eigene Transformationserfahrung und die doppelte Sozialisation sowohl in der DDR als auch im vereinten Deutschland geprägt sei, ist die Leitidee des Sammelbands. Trotz des damit verbundenen Versuches, diese Generation als politisches Subjekt zu konstituieren und ihr Gehör zu verschaffen, wird im Beitrag von Flack (2016) betont, dass diese Generation und ihre Erfahrungen heterogen seien und ihrer ostdeutschen Identität unterschiedliche Relevanz zuschreibe (ebd., S. 63f.). Der Vergleich beider Studien ermöglicht eine Intrakohortenstudie der Personengruppe, die zwischen 1975 und 1985 in der DDR geboren wurde. Zum ersten Untersuchungszeitpunkt 2004 waren die interviewten Mitglieder dieser Personengruppe zwischen 19 und 29 Jahren alt; zum zweiten Untersuchungszeitraum 2013-15 in der Studie von Flack (2016) waren sie etwa zehn Jahre älter (zwischen 28 und 40 Jahren). Mit diesem Intrakohortenvergleich unterschiedlicher Personen derselben Kohorte können sowohl Lebenszykluseffekte als auch Verschiebungen des gesellschaftlichen Diskurses verantwortlich für Veränderungen sein.

Der Studie von Kubiak (2018) liegen ebenfalls halbstrukturierte Befragungen in Form von Gruppendiskussionen zugrunde. Die Gruppendiskussionen wurden 2015/16 mit Studierenden in Berlin, Bremen, Rostock, Frankfurt am Main, Dresden, Köln und Leipzig geführt, wobei sich die Gruppen jeweils ausschließlich aus entweder ost- bzw. westdeutsch sozialisierten Studierenden zusammensetzten. Drei aufeinander folgende Themenblöcke, die jeweils durch Kubiak narrativ eingeleitet, aber darüber hinaus nicht strukturiert wurden, thematisierten Identität allgemein, deutsche Identität sowie west- bzw. ostdeutsche Identität (ebd., S. 33). Im Unterschied zur Studie von 2004 sind die Probanden bildungshomogen und wohnten zum Zeitpunkt der Befragung ausschließlich in Großstädten. Gruppendiskussionen bilden wie Einzelinterviews Identitätsaushandlungsprozesse ab, jedoch sind mehr als zwei Akteure involviert, weshalb eine multilaterale Konfrontation kennzeichnend für Gruppendiskussionen ist - allerdings in Abhängigkeit von der sozialen und politischen Homogenität der Gruppe mehr oder weniger ausgeprägt. In Bezug auf das Alter wurden bei Kubiak (2018) zwischen 1990 und 1995 Geborene untersucht, die zum Untersuchungszeitraum 2015/16 zwischen 20 und 26 Jahren alt waren und somit in der gleichen Phase ihres Lebenszyklus befragt wurden wie die 2004 interviewte Kohorte. Bei dem Vergleich der Ergebnisse von 2004 mit der Studie von Kubiak handelt es sich mithin um eine Interkohortenstudie, wobei sich die Kohor- 
ten durch die Geburtsjahrgänge und vor allem darin unterscheiden, ob sie eigene Erfahrungen in der DDR gemacht haben können. Hier können Verschiebungen auf Kohorteneffekte und Veränderungen des gesellschaftlichen Diskurses zurückzuführen sein.

Aufgrund dieser methodischen Unterschiede, die in der Interpretation berücksichtigt werden, handelt es nicht um Kohortenvergleiche strictu sensu, sondern um die Eröffnung potentieller Vergleichsperspektiven, die sich insbesondere dann ergeben, wenn trotz unterschiedlicher Methoden und divergierender theoretischer Hintergründe deutliche Parallelen in den Identitätskonstruktionen erkennbar sein sollten. Ausgangspunkt dieser Kohortenvergleiche ist die Inhaltsanalyse der in den Interviews von 2004 erfassten Identitätsbildungsprozesse. In Kontrastbildung wurden dabei sieben Typen ostdeutscher Identitätskonstruktionen identifiziert, deren Hauptmerkmale synoptisch in Abb. 1 dargestellt sind und die im folgenden Abschnitt im Detail erläutert werden.

Bei der vorgestellten Typologie ostdeutscher Identitätsbildung handelt es sich um die Verdichtung und Abstrahierung der empirischen Ergebnisse der Interviewstudie aus dem Jahr 2004. In Anlehnung an die Methode der typisierenden Strukturierung qualitativer Daten von Mayring (2015, S. 103 ff.) wurden die Ausprägungen ostdeutscher Identitätsbildung im Interviewmaterial paraphrasiert und strukturiert. Sowohl die insgesamt sieben Typen ostdeutscher Identitätsbildung als auch die Typisierungsdimensionen, die die schrittweise Kategorisierung der Typen ermöglichte, wurden „direkt aus dem Material in einem Verallgemeinerungsprozess ab[geleitet]“ (ebd., S. 85). Die induktiv entwickelten Typisierungsdimensionen sind in Abb. 1 jeweils als Kontinuum dargestellt, auf dem sich die einzelnen Typen positionieren lassen: 1) Bezug der Identitätsbildung (Identifizierung mit Ostdeutschland - Kategorisierung in Ost/West), 2) Zeitperspektive (Vergangenheit - Gegenwart), 3) Erklärung der Identitätsbildung (Kontextualisierung/Konstruktion - Naturalisierung) und 4) Art der Reaktion auf wahrgenommene Kategorisierung (Umdeutung - Internalisierung - Ablehnung).

\section{Typen ostdeutscher Identitäten im Jahr 2004}

\subsection{Abgrenzungsidentifizierer}

Personen des Typus „Abgrenzungsidentifizierer“ identifizieren sich im Interview selbst als ostdeutsch und bewerten diese Identität durchweg als positiv. Dies erfolgt entweder spontan oder die Fremddefinition durch den Interviewer als „ostdeutsch“ wird akzeptiert, wodurch die Salienz dieser kollektiven Identität unterstrichen wird. Charakteristisch für diesen Typ ist folgender Satz:

[...] na ja die [Brandenburger, L.V./J.L.] sind halt keine Wessis, oder? Die sind schon so drauf wie wir [Thüringer, L.V./J.L.]. Haben halt auch so ne Osterziehung, so wie wir. Also, die sind für mich genauso. (ID8)

Darin kommt zum Ausdruck, dass die größte Gemeinsamkeit zwischen Thüringern und Brandenburgern als Ostdeutsche - im Unterschied zu den Westdeutschen - 


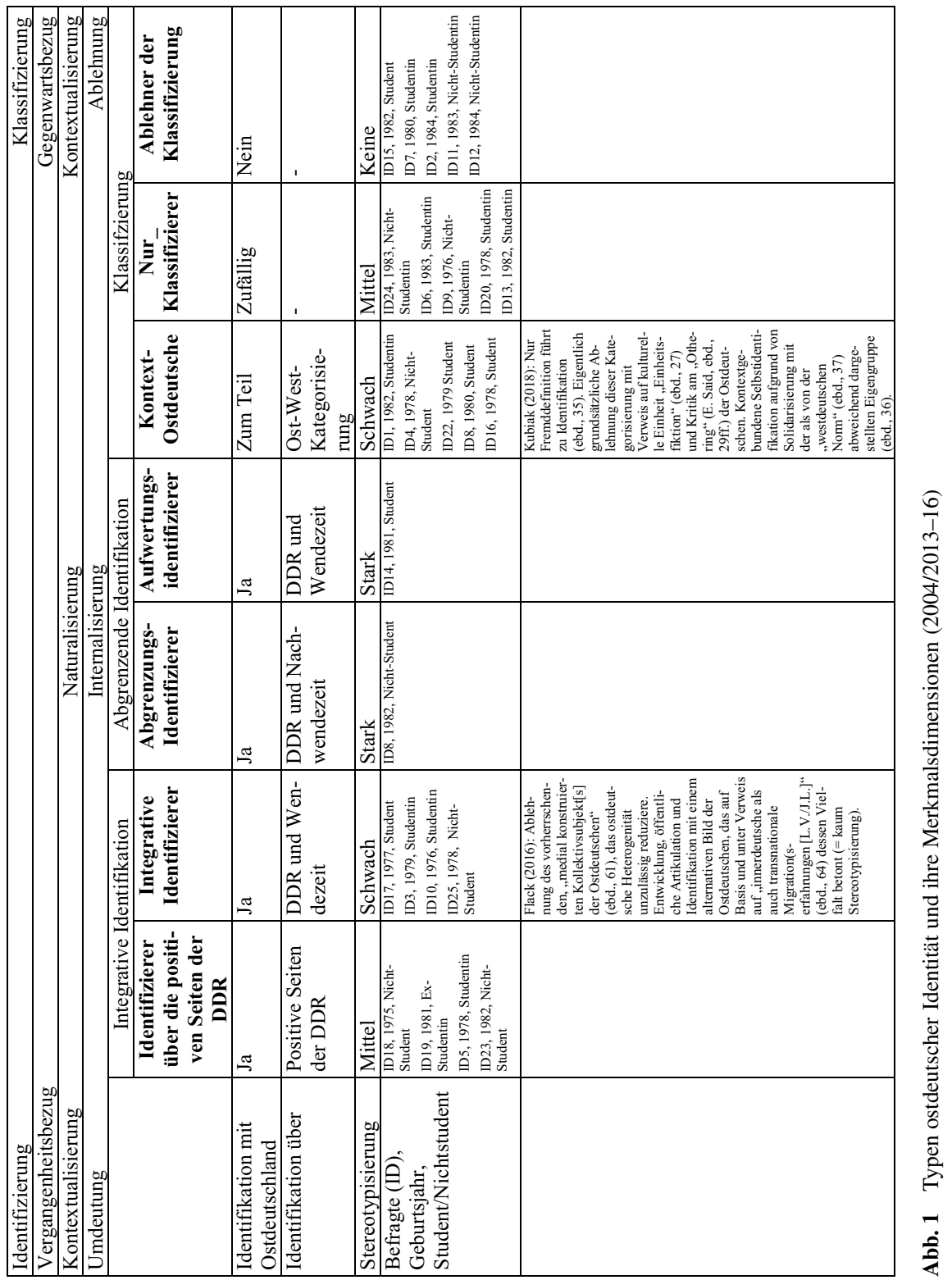


in der Sozialisation in der DDR gesehen wird. Die Betonung der Sozialisation dient der Naturalisierung der ostdeutschen Identität, denn damit wird impliziert, dass sich Gleichheit allein aufgrund der Zugehörigkeit zur Gruppe von selbst einstellen würde. Die Stereotypisierung der Ostdeutschen geschieht bei diesem Typus stets in Kontrastierung zu den Westdeutschen und wird inhaltlich als Unterschied zwischen individualistischen und kollektivistischen Grundorientierungen definiert. Diese Stereotypisierung bleibt auch bei gegenteiligen Erfahrungen im Kontext individueller Kontakte stabil:

Ähm, na in meiner Klasse gab es mal einen Wessi, der war aber nett, der hat ja auch im Osten gewohnt. (ID8).

Diese Stabilität wird erzeugt, indem der ,eine Wessi“ als „Westdeutscher im Osten" rekategorisiert wird, der damit durch den ostdeutschen Kontext geprägt wurde. Die ostdeutsche Identität dieses Typus speist sich zudem aus dem Gefühl der inferioren Position der Gruppe, die sich durch die wirtschaftliche Unterlegenheit ergebe (vgl. Brunner und Walz 1998):

[...] also wirtschaftlich gesehen schwach, wie alle Ostländer [...] Hier ist ja nach der Wende alles den Bach runtergegangen. [...] Ich bin ja schon länger arbeitslos [...] hab nichts Gescheites gekriegt. Das sieht im Osten echt mies aus gerade. (ID8)

In der Klassifizierung der Westdeutschen durch deren Heterostereotypisierung symbolisieren die Vertreter/-innen dieses Typus die Kompetenzen, die sie als notwendig für den Erfolg in der gegenwärtigen, als individualistischer wahrgenommen Gesellschaft ansehen. Damit können sie zugleich erklären, weshalb die Westdeutschen eine superiore Position einnehmen, da sie eher als die Ostdeutschen die notwendigen Eigenschaften für die individualistischere Gesellschaft besäßen. Eine positive Wertung der kollektiven Identität der Ostdeutschen wird dadurch erreicht, dass diese wirtschaftliche Vergleichsdimension zwar anerkannt, aber moralisch uminterpretiert wird. Die Westdeutschen seien zwar individualistischer, aber ,die sind alle viel arroganter als hier“ (ID8).

\subsection{Aufwertungsidentifizierer}

Die Legitimation der eigenen Identität und Sozialisation gelingt dem Typus der „Aufwertungsidentifizierer“ durch die Differenzierung der DDR in den ,schlechten Staat“" und die ,gute Gesellschaft“. Vertreter/-innen dieses Typus beziehen sich zugleich weniger auf eine ökonomische als auf eine wahrgenommene kulturelle OstWest-Asymmetrie. Sie beklagen z.B. den Wegfall der Ostprodukte und die Dominanz der Westprodukte:

Wir hatten eigentlich auch gute Produkte und irgendwie nicht schlechte, sondern im Gegenteil [...]. (ID14)

Zugleich wird die Dominanz Westdeutschlands in der Deutungshoheit über die Vergangenheit kritisiert. Somit ist diese Wahrnehmung die auffallendste Konsequenz bei diesem Typ, denn die DDR-Vergangenheit wird als mindestens gleich -, wenn 
nicht sogar höherwertig legitimiert - insbesondere dann, wenn sie als negativ bewertet wahrgenommen wird:

[...] ich denke, das kommt auch immer dann zum Vorschein, wenn man als Ossi präsentiert wird, also wenn man jetzt irgendwo in einer Gruppe steht und sich unterhält und dann plötzlich so: „Erzähl doch mal was aus dem Osten!“ Also das regt eigentlich erst Widerstand an und man sagt dann quasi: „Okay: Ja ich bin aus dem Osten und ich bin stolz draus, denn ich hab' eine gute Kindheit bekommen können.“ (ID14)

Eng damit verbunden ist eine Trennung in West- und Gesamtdeutschland, was einerseits auf den gesamtdeutsch integrierenden Aspekt dieses Typus ostdeutscher Identität hinweist, andererseits aber im Zusammenhang mit den oben genannten Aussagen zeigt, dass „Ost“ und „West“ in einer Art Wettbewerb um die besseren Eigenschaften empfunden werden. Ebenso wird nicht nur die Vergleichsdimension individualistisch/kollektivistisch umgewertet, sondern den Ostdeutschen werden auch mehr Eigenschaften auf der individualistischen Seite zugeschrieben, z. B. mehr Strebsamkeit. Die symbolische Konstruktion der Gleichheit der Ostdeutschen unterstellt ausgeprägte Homogenität im Inneren bei starken Unterschieden zu Westdeutschland.

[...] wenn du die ostdeutschen Jugendlichen ordentlich anleitest, die können sich über ganz andere Sachen [als die Westdeutschen] freuen, also mit denen kannst du, glaub' ich, wirklich noch in den Wald gehen und die können Gefallen daran finden [...] (ID14)

Wie die Abgrenzungsidentifizierer sehen auch die Aufwertungsidentifizierer die Sozialisation in der DDR als Ursache der ostdeutschen Identität. Allerdings nehmen sie auch Ursachen aus jüngerer Zeit wahr, z.B.:

Und [die ostdeutsche Identität] kam auch ein Stück weit durch die ganze Wende, dass dann halt viele aus dem Westen kamen, die ganzen Immobilienmakler und sich das unter den Nagel gerissen [haben] (ID14)

Zusammenfassend symbolisieren beide Typen durch die Artikulation ihrer ostdeutschen Identität eine von ihnen wahrgenommene ökonomische und kulturelle Asymmetrie zwischen Ost- und Westdeutschen, in der Ostdeutsche generell als abgewertet wahrgenommen werden. Da die Identifizierung beider Typen mit Ostdeutschland stark und positiv ist, reagieren sie darauf kollektiv durch die Abgrenzung zu den Westdeutschen und die Umdeutung des Gruppenverhältnisses mit dem Ziel, die wahrgenommene inferiore Stellung der Ostdeutschen entweder zu begründen oder die relevante Dimension des Vergleichs zu verändern und damit die Ostdeutschen aufzuwerten. Die Abgrenzung geschieht allerdings gegenüber Westdeutschland, nicht Gesamtdeutschland. Die mitunter auftauchende Nostalgie ist dementsprechend kein Wunsch nach einer Wiederherstellung der DDR als solcher, sondern nach Gleichberechtigung im vereinigten Deutschland. 


\subsection{Identifizierer über die positiven Seiten der DDR}

Die Angehörigen dieses Typus vertreten ebenso den Gedanken, dass ihre ostdeutsche Identität in der Sozialisation gründet, aufgrund derer sich Unterschiede in der „Mentalität“ zwischen Ost und West gebildet hätten: Man fühle sich ostdeutsch,

weil wir uns auch nach den ganzen Jahren immer noch ein bisschen von den Westdeutschen unterscheiden, denk' ich. Von der Mentalität her. (ID19)

Diese Unterschiede werden weniger als bei den beiden vorherigen Typen auf der Vergleichsdimension kollektivistisch/individualistisch verortet und falls doch, werden sie in geringem Ausmaß moralisch umgewertet. Relevanter sind hingegen wahrgenommene Unterschiede in Eigenschaften wie „Improvisationstalent“ und „Naturverbundenheit“, wobei auffallend ist, dass es den Vertreter/-innen dieses Typus Schwierigkeiten bereitet, Unterschiede und Gemeinsamkeiten konkret zu benennen:

[...] die sind alle ein ähnlicher Menschenschlag wie ich, ich kann Dir hier und jetzt nicht sagen, inwieweit und warum. (ID5).

Die symbolische Konstruktion interner Homogenität ist bei diesem Typ weniger konkret. Es wird zudem eine interne Heterogenität der Gruppen eingeräumt, womit Deklassifizierung stattfindet und eine Reklassifizierung stattfinden kann:

Es gibt mit Sicherheit auch Ostdeutsche, mit denen man nicht klarkommt [...] (ID18)

Es gibt solche und solche. Aber wenn ich an einen Wessi denke, dann ist das immer noch manchmal ein bisschen [Hervorhebung L.V./J.L. zur Verdeutlichung der relativierten Stereotypisierung] negativ (ID19)

Damit verbunden lässt dieser Typus auch die Wirkung der Sozialisation nicht unerklärt:

[...] bei uns ist die [...] Freundschaft unter den Menschen [...] vielmehr vorhanden [...]. Das ist in dem anderen Teil Deutschlands und in der jetzigen Jugend nicht mehr so, weil [...]. Die haben doch alles gehabt und waren auf niemanden angewiesen. Bei uns, ohne Freunde warst du aufgeschmissen. (ID18)

Dieser Typus erachtet die Gemeinsamkeiten nicht als natürlich, sondern führt sie auf die gesellschaftliche Struktur der DDR zurück. Diese Kontextualisierung stellt im Vergleich zu den Typen der Abgrenzungs- und Aufwertungsidentifizierer eine Relativierung ihrer Bedeutung dar. Die heutige gesellschaftliche Situation sei folglich nicht geeignet, diese ostdeutschen Eigenschaften fortbestehen zu lassen:

[...] [ich denke] also es hebt sich jetzt auf jeden Fall auf, mein Bruder zum

Beispiel, der ist 20, der hat das nicht mehr so wie wir [...] (ID5)

Dies zeigt, dass das Verhältnis zwischen Ost- und Westdeutschen nicht als Ursache für eine ostdeutsche Identität gesehen wird, mithin sich Ost- und Westdeutsche für diesen Typus nur auf einer relativ unproblematischen mentalen Ebene unterscheiden würden: 
Ich denk' das Problem sind wirklich die Vorurteile (ID19)

Das ist schon ein besonderes Gefühl, dass man nicht unbedingt etwas Besseres hat, aber etwas Eigenes. Das grenzt schon ab, wenn auch auf einem bisschen humorvollen Weg. (ID23)

Zudem setzen sich die Vertreter dieses Typus in eine kognitive Distanz zur DDR. Dies gelingt ihnen weitgehend damit, dass sie sich als zu jung deklarieren, um wirklich etwas von der DDR und insbesondere von ihrer politischen Dimension erfahren zu haben. Es erfolgt eine Trennung in Staat und Gesellschaft:

[...] also [die DDR, L.V./J.L.] ist auf jeden Fall ne Diktatur gewesen, die viel schlechte Eigenschaften hatte [...] es gab aber auch positive Seiten, was jetzt zum Beispiel Familie und Freunde betrifft [...] (ID5)

Dieser Typus erklärt, die DDR als Staat kaum erlebt zu haben und deshalb:

Ich kann mich an nichts Negatives aus der DDR erinnern. (ID19)

[...] denn als Kind [...] war die DDR schön. (ID18)

In dieser Darstellung doppelter Differenz - zum einen zu den heutigen Jugendlichen, die die DDR gar nicht mehr erlebt haben, und zum anderen zu den negativen Verhältnissen in der DDR, die man nicht erfahren habe und sich deshalb damit auch nicht auseinandersetzen müsse - liegt der Kern zum Verständnis dieses Identitätstypus, denn mit der kognitiven Distanzierung ergibt sich die Möglichkeit, das Identitätsangebot „ostdeutsch“ als eine bloße Kategorisierung ohne Rückgriff auf damit verbundene Erfahrungen und Wissensbestände aus der DDR zu definieren. Folglich ist die symbolische Konstruktion von Gleichheit auffallend inhaltsarm und dieser Typus kann sich mit einer Vielzahl heterogener individueller Eigenschaften einfacher auf das Identitätsmodell „ostdeutsch“ beziehen.

Zusammenfassend ist dieser Typus eher integrierend als abgrenzend. Für diesen Typ soll die ostdeutsche Identität in eine gesamtdeutsche Identität eingebracht werden, ohne sich allzu stark von den Westdeutschen abzugrenzen. Dafür spricht auch, dass die Wiedervereinigung positiv bewertet wird:

[...] also eigentlich muss man dem Ganzen [der Wiedervereinigung, L.V./J.L.] auch total dankbar sein, sonst wären wir auf keinen Fall da, wo wir heute sind. (ID5)

Die Distanzierung von den Jugendlichen, die keine ostdeutsche „Mentalität“ mehr besäßen, ist zugleich eine Kritik an den wahrgenommenen gegenwärtigen gesellschaftlichen Verhältnissen, denen die als positiv erinnerten Seiten der DDR entgegengestellt werden, insbesondere die eher gemeinschaftlich strukturierten Beziehungen. Diese Kontrastierung erinnert an die Vergleichsdimension kollektivistisch/ individualistisch. Sie wird allerdings für den intertemporalen Vergleich zwischen der DDR und dem heutigen Deutschland gebraucht und weniger als Intergruppenvergleich zwischen Ost- und Westdeutschen. 


\subsection{Integrative Identifizierer}

Der Typ der Integrativen Identifizierer ist in seinen Einstellungen heterogener als die vorherigen Typen. Vertreter/-innen dieses Typus ist jedoch gemeinsam, dass sie explizit oder implizit bekunden, die DDR und insbesondere die Wendezeit bewusst miterlebt zu haben:

Dann habe ich diese ganze Wendegeschichte und auch teilweise die DDR schon noch mitgekriegt [...] (ID3)

Unter den Vertreter/-innen dieses Typus findet sich keine Person, die nach 1979 geboren wurde. Die Erfahrung der Wende wird als Bruch wahrgenommen:

[...] weil da [im Film „Good Bye Lenin“, L.V., J.L.] eben dargestellt wird, wie die Leute wirklich damit umgehen, [mit] diese[m] Bruch [...] (ID10)

Aus dieser plötzlichen und krisenhaften Veränderung bisher geltender Deutungsund Handlungsorientierungen durch das Ende der DDR erwächst für die Integrativen Identifizierer die Notwendigkeit, die Erfahrungen sowohl aus der DDR- als auch der Wendezeit in die eigene Identitätskonstruktion einzubeziehen. Damit unterscheiden sie sich von den Typen, deren kognitive Distanzierung von der DDR diese Notwendigkeit verhindert. Die Selbstidentifizierung als „ostdeutsch“ wird zwar teilweise über die Erfahrung der Sozialisation begründet, aber vorrangig wird auf die Erfahrung der Wendezeit Bezug genommen:

Eindeutig nach der Wende. Also gerade die Wendezeit und danach waren die prägenden. (ID10)

Dieser Bezug ist so zu deuten, dass der Begriff „Wendezeit“ den Übergang des alten Systems in das neue am prägnantesten symbolisiert. Die symbolische Konstruktion von Gleichheit erfolgt über das Symbol der „Wende“ ${ }^{\text {“3 }}$. Die gemeinsam geteilte Erfahrung der „Wende“ wird, was immer sie für den Einzelnen bedeutete, für die Ostdeutschen als Negativbewertung ihres Lebens bis dahin gedeutet, die sich aber bei erfolgreicher Identitätskonstruktion als eigentlich positiv herausstellt:

Denn ich bin aus dem Osten und finde das eigentlich auch nicht schlimm. Im Gegenteil - aber es ist schwer da so ein positives Bild zu entwickeln. Also mittlerweile wird das besser, weil der Systemwandel auch positiver bewertet wird für die jungen Leute damals. Also man sagt, man wird viel flexibler sein, man kennt zwei Systeme, also man beweist, dass man da flexibel ist. (ID10)

„Eigentlich“ im Zusammenhang mit ,aber es ist schwer“ impliziert, dass die Interviewten mit ihrer Selbstdefinition als ostdeutsch häufig auf vorherrschende Fremddefinitionen stoßen, die eine solche Identifizierung negativ beurteilen. Der Ausweg zur Erhaltung einer positiven Identität ist eine Umdeutung des als vorherrschend wahrgenommenen Identitätsangebots „ostdeutsch“ mittels eigener biographischer Sinngehalte. Die Erfahrung, sowohl die DDR als auch die heutige Gesellschaft zu

\footnotetext{
${ }^{3}$ Dass dieser Begriff von Egon Krenz mit dem Ziel der Aufrechterhaltung der sozialistischen Ordnung in der DDR eingeführt wurde, spielt in keiner der Aussagen eine Rolle.
} 
kennen, wird positiv gedeutet, da sie Flexibilität ermöglicht - eine Eigenschaft, die in der gegenwärtigen individualistischen Gesellschaft als notwendig erachtet wird. Dies gilt jedoch nur, wenn man sich auch in der heutigen Gesellschaft zurechtfinde, d.h. zumindest eine konsistente, zwei unterschiedliche gesellschaftliche Systeme integrierende Identität konstruieren kann:

Und das jetzt als Ostdeutsche, das ist fast so als ob ich jetzt ... meine Identität ist wie ein Puzzle eigentlich ... oder wie so eine Kinderüberraschung, die aus zwei Teilen besteht. Und man nimmt jetzt das Positive, oder das, was man selbst als positiv erachtet, was in der DDR o.k. war, das versucht man irgendwie mitzunehmen, jetzt in eine Zeit, die wiederum andere Vorteile hat, aber auch Nachteile [...] (ID10)

Vor diesem Hintergrund versteht dieser Typ die Integration der ostdeutschen Identität in eine gesamtdeutsche als normativ gefordert. So ist die Autostereotypisierung der Ostdeutschen davon geprägt, dass vielen Ostdeutschen die Flexibilität und Anpassungsfähigkeit fehle:

Klar, Ossis sind auch furchtbare Bärmler, dass es ihnen so schlecht geht in der neuen Zeit. Klar, kann schon sein, dass es ihnen schlecht geht, aber was haben sie sich denn erwartet? Manchmal wirken sie auch ein bisschen schafköpfig, weil sie halt noch nicht mitgekriegt haben, dass jetzt alles ganz anders ist und dass die Werte von damals nicht mehr zählen. (ID17)

[...] viele Leute sind da halt noch nicht so weit, dass die halt das ostdeutsche Denken haben [gemeint ist: das westdeutsche Denken, L.V./J.L.] und das merkt man schon [...] manche Leute natürlich nicht, viele haben den Sprung schon geschafft. (ID25)

Die Vergleichsdimension kollektivistisch/individualistisch wird pragmatischer als in den anderen Gruppen gehandhabt. Sie wird zwar moralisch umgewertet und als Erklärung für ökonomische Unterschiede verwendet:

[...] dieser Kampf so in dem neuen System, der fällt mir schon schwer. [...] Wenn man dann aufgewachsen ist, in einem System wo wir Solidarität hatten [...] und jetzt ist das extrem auf Leistung fokussiert und Konkurrenz [...] Da merke ich einfach, da unterscheide ich mich, weil ich da oft auch naiv noch einfach bin, aber auch zu weich bin. (ID10)

Sie wird jedoch zugleich mit einer Forderung an die Ostdeutschen verbunden, sich entlang individualistischer Werte umzuorientieren:

[...] meines Erachtens wird viel zu wenig dagegen gemacht, gegen dieses ostdeutsche Klischee. Man kann das ja versuchen abzubauen oder so, das ist nicht immer nur, dass die Wessis das probieren, das sind ja normalerweise auch die Ossis, die müssten ja normalerweise auch, sagen nee, wir vermarkten uns jetzt so dermaßen gut, dass es keine Unterschiede mehr gibt. (ID25)

Damit ist eine Kritik an denjenigen Ostdeutschen verbunden, die sich noch nicht an die neuen Verhältnisse angepasst hätten. Zugleich zeigen die Interviews die Schwierigkeiten einer solchen Anpassung, die auch darin gesehen werden, dass das 
Thema der ostdeutschen Identität und der DDR-Vergangenheit nicht im kulturellen Diskurs auftaucht:

Einfach weil die Geschichte finde ich jetzt ... total wird das totgeschwiegen. Man redet kaum mehr. (ID10)

Die DDR wurde einfach wieder eingegliedert, die wurde einfach zusammengeführt ... es wurde einfach das BRD-Modell übernommen, es gab minimale Anpassungen ... minimale [...] (ID10)

Die Vertreter/-innen dieses Typus finden sich somit in einer ambivalenten Haltung zur Gruppe der Ostdeutschen. Einerseits fühlen sie sich zugehörig und sehen das zentrale Autostereotyp der fehlenden Anpassungsfähigkeit für einen großen Teil der Ostdeutschen als bestätigt an. Andererseits lehnen sie dieses zentrale Stereotyp für sich selbst ab und konstituieren damit eine Person/Gruppe-Diskrepanz, d.h. sie vergleichen sich als Person und als Gruppenmitglied auf verschiedenen Dimensionen, weshalb die eigene Lage nicht zwingend mit der Selbstkategorisierung als Gruppenmitglied zusammenhängen muss (vgl. Mummendey und Kessler 2000, S. 282). Funktionaler Kern der ostdeutschen Identität ist hier nicht die Aufwertung der Ostdeutschen als Gruppe, sondern die Anerkennung der eigenen, individuellen Vergangenheit und der eigenen Integrationsleistung.

\subsection{Kontext-Ostdeutsche}

Während die Integrativen Identifizierer deutlich zum Ausdruck brachten, dass sie sich als Ostdeutsche fühlen, sind die Vertreter/-innen des Typus der Kontext-Ostdeutschen zurückhaltender:

„Als Kind fühlst du dich halt als DDR-Bürger, deshalb war es auch kein Problem zu sagen, ich bin jetzt plötzlich Bundesbürger.“ - INT: „Aber Du fühlst Dich eher als Deutscher, nicht als Ossi?“ - „Ja klar, auf jeden Fall, es sei denn, der Andere sieht sich als Wessi. Wenn der das trennt, dann trenn ich das auch für mich, dann seh' ich mich demgegenüber auch als Ossi ... Aber sonst nicht, sonst seh' ich mich in erster Linie als Deutscher.“ (ID22)

Hier steht die (wechselseitige) Klassifizierung und weniger die Identifizierung im Vordergrund. Wahrgenommene Unterschiede werden zwar auch auf die Sozialisation zurückgeführt, aber die Identifizierung findet primär dann statt, wenn OstWest-Unterschiede in Fremddefinitionen durch andere artikuliert werden. Allerdings werden diese Unterschiede als bedeutungsvoll akzeptiert, denn die Selbstdefinition der Westdeutschen als Westdeutsche wird nicht durch Re- oder Deklassifizierung als bedeutungslos dargestellt. Für diesen Typus gewinnen Unterschiede zwischen Ost- und Westdeutschland somit erst dann eine Bedeutung, wenn sie durch andere artikuliert und damit als bedeutend definiert werden:

INT: „Nun fühlen sich aber Jugendliche, die ich kenne, immer noch als Ossis, obwohl sie gar nicht oder nur kurz die DDR-Zeit miterlebt haben. Woran liegt das?“ - „Na an den Eltern oder an den Medien, weil die das halt immer noch 
trennen, sagen da ist das und da ist das in den alten und den neuen Bundesländern." (ID22)

Aber auch Ostdeutschland, weil es in der letzten Zeit so stark thematisiert wird. (ID1)

Dieser Typus thematisiert die Kontextabhängigkeit der Selbstidentifizierung als ostdeutsch, um zu verdeutlichen, dass ihnen weder die Ostidentität noch die Klassifizierung nach Ost und West wichtig ist:

$\mathrm{Na}$ ja, das mit dem Ost-West sagt man eher so zum Spaß. Man macht halt seine Witze drüber, aber ansonsten habe ich eigentlich nicht so viele Vorbehalte.

(ID4)

Oder:

INT: „Du würdest nicht sagen, dass du aus Ostdeutschland kommst?“ -,Nö.“ (ID1) $)^{4}$

Identifizierung folgt also (nicht nur bei diesem Typus) aus der Klassifizierung, wenn sie von Personen mit Deutungsmacht als relevant dargestellt wird und das scheinen vor allem die Westdeutschen zu sein. ${ }^{5}$ Allerdings muss angemerkt werden, dass die Einordnung einer Person aufgrund eines bestimmten Verhaltens - ,wenn der Andere sich als Wessi sieht" - eine Fremddefinition durch den Interviewten ist. Wenn damit eine Selbstdefinition als „ostdeutsch“ korreliert, geht die Klassifizierung auch von den Interviewten selbst aus - entgegen der eigenen Wahrnehmung dieses Typus. Diese Vermutung wird unterstützt, weil diejenigen, die konkret erklären, sie haben bereits die Selbstklassifizierung von Westdeutschen erfahren, konkrete Autound Heterostereotype nennen:

Überall wo man hingeht in Ostdeutschland, findet man auch die Menschen mit diesen Eigenschaften [gemeint sind Herzlichkeit, Zusammenhalt, Unterstützung, L.V./J.L.], während es ,drüben“ eher schwer ist, so etwas zu finden. (ID8)

Diejenigen, die keine Selbstklassifizierung der Westdeutschen als Erfahrung nennen, sprechen dagegen nur im Allgemeinen von einer Trennung:

„Für mich gibt's schon auch die Unterscheidung zwischen Ost und West [...]“ - INT: „Und wie genau bestimmt sich für dich ein Wessi?“ - ,[...] Ohh ... kann ich nicht so allgemein sagen ... kann man schlecht unterscheiden ... der Wessi ist halt vielleicht rhetorisch besser in der Lage als der Ossi, manchmal, aber das liegt halt an der anderen Vorprägung.“( (ID22)

\footnotetext{
4 Bei dieser Interviewten wird bei dem vorherigen Zitat klar, dass sie sich nicht abgrenzen will, sondern dazu getrieben wird. Die Antwort auf die erste Frage wurde mit dem ,weil es [...] thematisiert wird“ begründet und bei der zweiten Frage nach dem Verhalten gegenüber Westdeutschen lehnt sie die Identifikation als Ostdeutsche ab, möglicherweise aufgrund des Abgrenzungscharakters.

5 Allerdings zeigt sich auch, dass es die Ostdeutschen sein können, z. B. wenn der (ostdeutsche) Interviewer als Person mit Deutungsmacht wahrgenommen wird.
} 
Die Stereotypisierungen sind jedoch weniger ausgeprägt als bei den bisher beschriebenen Typen. Insbesondere im Vergleich zum Abgrenzungsidentifizierungstypus fällt auf, dass die persönliche Begegnung als Anlass wahrgenommen wird, Heterostereotype gegenüber der anderen Gruppe abzubauen:

[...] wenn man die [die Westdeutschen] erst einmal personifiziert hat, dann sind die Vorurteile weg. (ID1)

Zusammenfassend lässt sich diese Gruppe mit dem Satz charakterisieren:

Also das Ganze ist schon noch im Kopf drin. (ID4)

Damit kommt zum Ausdruck, dass die Klassifizierung zwar als überflüssig empfunden wird, weil es keine relevanten Unterschiede gebe, sie aber dennoch wirksam ist. Diese Wirksamkeit wird jedoch gleichzeitig bedauert. Dieser Typus identifiziert sich nur dann als „ostdeutsch“, wenn der Ost-West-Unterschied in seiner Wahrnehmung situativ als bedeutsam definiert wird. Die Identifizierung ist daher eher mit konkreten individuellen Ereignissen als Gruppenmitglied als mit dem wahrgenommenen kollektiven Status der Ostdeutschen als Gruppe verknüpft. Da diese eigenen Erfahrungen vielfältiger sind als die durch Stereotype symbolisierte Intergruppenbeziehung zwischen Ost- und Westdeutschen, ist auch die Heterogenität innerhalb der Einstellungen in dieser Gruppe ausgeprägter.

\subsection{Nur-Klassifizierer}

Dieser Typus identifiziert sich nicht als ostdeutsch. Vielmehr distanzieren sich die Interviewten von einer Identifizierung über diese Klassifizierung:

INT: „Und, spielt das [Unterschiede zwischen Ost- und Westdeutschland, L.V./J.L.] für dich eine Rolle, begreifst $\mathrm{Du}$ dich in manchen Situationen als Ostdeutsche?“ - „Also, da fällt mir jetzt spontan keine Situation ein.“ (ID9) Also, diese Einteilung [Ost/West, L.V./J.L.] spielt für mich eigentlich keine Rolle. Irgendwie gehört es nicht zu meiner Identität. (ID24)

Vertreter/-innen dieses Typus kategorisieren trotzdem nach Ost- und Westdeutschen und machen ihre Distanzierung davon daran deutlich, dass sie in der dritten Person von den Ostdeutschen sprechen:

Das ist eben der Ossi, wo er sich erkennt. [...] Also manchmal habe ich das Gefühl, dass Ossis mehr selber machen [...] (ID6)

Die Klassifizierung wird zwar ebenso auf Basis von Eigenschaften vorgenommen, die der Vergleichsdimension kollektivistisch/individualistisch zugeordnet sind. Diesen Eigenschaften und Unterschieden wird jedoch nur eine geringe Bedeutung zugeschrieben:

Da ist nicht soviel Zusammenhalt zum Beispiel. Ich betrachte das aber weniger wertend. Es war einfach da und deswegen bin ich in einer anderen Generation, als die nach uns kommt zum Beispiel. (ID20) 
Die Interviewten benennen auch häufig konkrete wirtschaftliche Unterschiede zwischen Ost- und Westdeutschland:

[...] dass sich Leute, die sich von Ostdeutschland in Westdeutschland bewerben, weniger Chancen haben als Westdeutsche. (ID9)

Dass vielleicht die Westdeutschen ein großes Auto fahren können, weil eben der Reichtum schon sehr ungleich verteilt ist. Und dass dort viele Häuser haben und hier im Osten viele im Neubaublock wohnen, obwohl man sagen muss: Wer wohnt heute noch im Neubaublock? (ID24)

Allerdings findet keine Begründung der wirtschaftlichen Asymmetrien über die unterschiedlichen Eigenschaften der Ost- und Westdeutschen statt, denn es wird deutlich gemacht, dass Unterschiede nur für die ,ältere Generation“ eine Rolle spielen würden:

INT: „Du sagst trotzdem, diese Grenzziehung zwischen Ost und West gibt es eigentlich nicht, weil diese Trennung ja nicht der Wirklichkeit entspricht.“ „Na ja, die Trennung in Ost und West nicht in meiner Generation, aber durchaus für die Elterngeneration, für die schon.“"(ID24)

Die Kombination aus ungleich wahrgenommenen Wirtschaftsverhältnissen mit der Ablehnung der Identifizierung als „ostdeutsch“ und der fehlenden Relevanz der Klassifizierung deutet auf eine wahrgenommene Durchlässigkeit des Statusverhältnisses zwischen Ost und West hin. Die Interviewte ID9 sagt beispielsweise, dass Ostdeutsche geringere Chancen in Westdeutschland hätten, aber sie führt weiterhin aus, dass sie zugleich die Erfahrung gemacht hätte, dass

[du dich] dann bemühen [musst], halt so gut wie möglich ... nicht so sächsisch ... zu reden [...]. (ID9)

Dieser Typus bevorzug die Strategie persönlicher sozialer Mobilität, indem die Klassifizierung zwar anerkannt wird, aber die Zuordnung zur Gruppe der Ostdeutschen wenig relevant ist, weil man sie individuell verlassen kann und sie damit unwirksam wird.

\subsection{Ablehner der Klassifizierung}

Die Vertreter/-innen dieses Typus vollenden die Tendenz, in ihrer Identitätsbildung primär die Klassifizierung zwischen Ost- und Westdeutschland vor Augen zu haben. Weil sie diese Klassifizierung ablehnen, identifizieren sie sich auch nicht mit Ostdeutschland. Dies geschieht auf zwei Arten. Einerseits gibt es diejenigen, die die Klassifizierung nach Ost- und Westdeutschland für nicht existent halten und sich deshalb nicht als Ostdeutsche identifizieren:

[...] ich würde mich nicht als Ostdeutsche bezeichnen und wenn, dann, dann werde ich einfach nur da rein gedrückt, weil ich in den neuen Bundesländern wohne und hier geboren bin. (ID2)

Andererseits gibt es diejenigen, die die Klassifizierung ebenso ablehnen, sich aber zu Ostdeutschland zugehörig fühlen, wenn eine Fremdklassifizierung als ,ost- 
deutsch" vorgenommen wird. Im Gegensatz zu den Kontext-Ostdeutschen dient die Artikulation dieses Zugehörigkeitsgefühls jedoch dazu, die Klassifizierung abzulehnen und zum Ausdruck zu bringen, dass Ostdeutschland und die Ostdeutschen nicht anders seien als Westdeutschland und die Westdeutschen:

Wenn ich immer darüber höre, nervt mich eine Sache. Wir sind ein vereinigtes Deutschland und wenn so herablassend über die Ostdeutschen geredet wird. Die Ossis wären blöd und so weiter. Ich versuche auch den Osten mit zu verteidigen, weil ich ja hierher gehöre. (ID12)

Es gibt diese Stereotypen, aber ich denke, dass die Menschen im Grunde alle gleich sind. (ID11)

Insgesamt äußern die Vertreter/-innen dieses Typus die Ansicht, dass sie sich nicht mit Ostdeutschland identifizieren können, weil sie die DDR selbst kaum erlebt hätten.

Ja, ich bezeichne mich als Deutsche und fertig. Weil dadurch, dass ich bei der Wende sechs Jahre alt war, warum bin ich dann ostdeutsch? Ich war ja noch nicht mal Pionier. (ID2)

Weil ich eben diese Verlängerung oder diese vermeintliche ostdeutsche Identität auch als Verlängerung der DDR betrachte. Da ich die DDR insgesamt, wie ja gehört, negativ werte, kann ich mit dieser Identität des Ostdeutschen wenig anfangen. (ID15)

Die Möglichkeit für eine ostdeutsche Identität wird folglich ausschließlich in der eigenen Erfahrung der DDR gesehen. Da es weder diese noch wahrgenommene Unterschiede zwischen Ost- und Westdeutschen gebe, sehen sie keine Möglichkeit der Identifizierung oder der Klassifizierung. Allerdings ist diese Ablehnung eher als eine normative Zurückweisung zu verstehen als eine empirische Feststellung, denn typischerweise wird wahrgenommen, dass Andere Ost-West-Klassifizierungen vornehmen. Nur eine der Interviewten gibt an:

[...] ich merke keinen Unterschied in der Behandlung [...] und ich behandle die Leute auch nicht, als ob sie ostdeutsch oder westdeutsch wären. (ID7)

Die Vertreter/-innen dieses Typus kategorisieren selbst nicht nach Ost-West und zeigen deshalb in den Interviews keinerlei Hetero- oder Autostereotypisierungen. Auch das DDR-Bild dieser Gruppe ist - mit einer Ausnahme - auffallend inhaltsarm:

INT: „Gibt es negative Sachen, die du mit Ostdeutschland verbindest?“ - „Ich weiß halt nicht viel über die DDR.“- INT: ,Ich meine jetzt Ostdeutschland.“Schweigen und Achselzucken (ID12)

Nein, überhaupt nicht, gar nicht, auch ich muss ehrlich sagen, ich kann mich an viele Sachen gar nicht erinnern, sondern ich sehe es jetzt in diesem Fernsehshows und muss darüber lachen, weil es so war. (ID7)

Es ist für diesen Typus auch nicht relevant, sich mit der DDR zu beschäftigen, da die bewusst wahrgenommene eigene Biographie erst später beginne:

Mir kommt, wie Helmut Kohl wahrscheinlich sagen würde, die Gnade der späten Geburt zu Gute. (ID15) 


\section{Die Heterogenität ostdeutscher Identitäten im Jahr 2004}

Jenseits der geschilderten und in Abb. 1 zu einer dimensionalen Darstellung verdichteten Unterschiede zwischen den Typen bestehen Gemeinsamkeiten, die auf ein kollektiv geteiltes Identitätsangebot hinweisen. Selbst diejenigen, die sich nicht als „ostdeutsch“ identifizieren oder eine solche Klassifizierung ohnehin ablehnen, beziehen sich letztendlich darauf, indem sie dieses Modell für sich selbst oder insgesamt zurückweisen. Nach diesem Identitätsangebot werden Ostdeutsche als Personen wahrgenommen, die a) selbst in der DDR aufgewachsen sind, ${ }^{6}$ die b) auf der zentralen Vergleichsdimension kollektivistisch/individualistisch eher auf der Seite des kollektivistischen Handelns einzuordnen sind und diese Einordnung auch dazu dient, die wahrgenommene kulturelle oder ökonomische Inferiorität der Ostdeutschen zu erklären und c) die sich aufgrund dieser Unterschiede selbst als ostdeutsch identifizieren oder zumindest die entsprechende Kategorisierung akzeptieren.

Jedoch reagieren die einzelnen Typen unterschiedlich auf dieses kollektiv geteilte Identitätsangebot. Die Abgrenzungs- und Aufwertungsidentifizier übernehmen dieses Modell und naturalisieren es als Reaktion auf die von ihnen wahrgenommene ökonomische oder kulturelle Inferiorität der Ostdeutschen im Vergleich zu den Westdeutschen. Um in dieser Situation den Gruppenvergleich positiv ausfallen zu lassen, grenzen sie sich durch extensiven Rückgriff auf Auto- und Heterostereotypen von den Westdeutschen ab und deuten das Gruppenverhältnis um, mit dem Ziel, die inferiore Stellung der Ostdeutschen zu begründen oder die Ostdeutschen aufzuwerten. Die starke Identifizierung mit Ostdeutschland bei gleichzeitiger kollektiver Aufwertung der Eigengruppe dient zugleich dazu, die individuelle Biografie und Identität aufzuwerten. Durch die Naturalisierung der Ost-West-Unterschiede werden beide Gruppen auch als wenig durchlässig wahrgenommen, wodurch die gewählte kollektive Strategie der Umdeutung des Gruppenverhältnisses die plausibelste Reaktion ist.

Weder für die Identifizierer über die positiven Seiten der DDR noch für die Integrativen Identifizier steht die Abgrenzung gegenüber Westdeutschland im Vordergrund ihrer ostdeutschen Identitätsbildung. Sie identifizieren sich vorrangig über die individuellen positiven Erfahrungen mit und in der DDR oder über die Erfahrung der Bewältigung des Systemumbruchs, mit der eine spezifische Transformationskompetenz einhergehe (vgl. Lettrari et al. 2016). Dieser Typus ostdeutscher Identität lehnt das kollektive Identitätsangebot „ostdeutsch“ für sich selbst ab, sieht es jedoch insbesondere für die ältere Generation als zutreffend an. In dieser Distanzierung kommt die individuelle Strategie zum Ausdruck, die eigene Identität in eine transformierte gesamtdeutsche oder transnationale Identität einbringen zu können. Der Versuch der Transformation des kollektiven Identitätsangebots ,deutsch“ geschieht über die Klassifizierung der (jüngeren) Ostdeutschen, die aufgrund ihrer spezifischen Erfahrungen Eigenschaften mitbringen, die gewinnbringend in die individualistische,

\footnotetext{
${ }^{6}$ Zum Befragungszeitpunkt 2004 stellte sich die Frage erst in Ansätzen, ob die ostdeutsche Identität bei denen entstehen kann, die kurz vor oder erst nach 1989 auf dem Gebiet der ehemaligen DDR geboren und sozialisiert wurden. Diese Personen waren zum Interviewzeitpunkt 2004 höchstens 14 Jahre und dürften daher in entsprechenden Diskursen weder aktiv noch passiv thematisiert worden sein.
} 
globalisierte Gesellschaft eingebracht werden können. Diese individuelle Strategie der Reklassifizierung ist integrativ und nicht abgrenzend, da sie die ostdeutsche Identität als Ergänzung der gesamtdeutschen/transnationalen Identität und als Mittel zur individuellen Überwindung der ohnehin als durchlässig wahrgenommenen Gruppengrenze ostdeutscher Identität versteht.

Die Kontext-Ostdeutschen, die Nur-Klassifizierer und die Ablehner der Klassifizierung nehmen das kollektive Identitätsangebot „ostdeutsch“ fast ausschließlich in Situationen wahr, in denen sie durch Fremddefinitionen selbst als ostdeutsch klassifiziert werden oder die Klassifizierung Ost-West allgemein als relevant dargestellt wird. Für sie selbst sind weder die ostdeutsche Identität noch die Ost-West-Klassifizierung relevant für ihre Handlungsorientierungen. Auf diese Situationen wird unterschiedlich reagiert: Kontext-Ostdeutsche identifizieren sich in diesen Situationen als ostdeutsch, die Nur-Klassifizierer und Ablehner der Klassifizierung tun dies wiederum nicht. Die Nur-Klassifizierer gestehen zwar fortbestehende Ost-West-Unterschiede zu, insbesondere für die ältere Generation, sie selbst identifizieren sich jedoch nicht als ostdeutsch und nehmen deshalb in Anspruch, dass diese Zugehörigkeit weder in ihren Selbst- noch in den an sie gerichteten Fremddefinitionen eine Rolle spiele. Die Gruppengrenze wird daher als durchlässig wahrgenommen und als Reaktionsstrategie wird folglich individuelle Mobilität gewählt. Die Ablehner der Klassifizierung lehnen sowohl die Identifizierung als auch die Ost-West-Klassifizierung generell ab und reagieren auf entsprechende Fremddefinitionen mit der Negation der Ost-West-Unterschiede unter Verweis auf die interne Heterogenität beider sozialer Kategorien. Diese drei Typen betonen deshalb die Kontextabhängigkeit ihrer Identitätskonstruktionen, während die übrigen Typen eine Unabhängigkeit ihrer ostdeutschen Identität von den Kontexten ihrer sozialen Interaktionen in Anspruch nehmen.

\section{Ostdeutsche Identitäten ein Jahrzehnt später: Perspektiven für einen Intra- und Interkohortenvergleich}

Die geschilderten Ergebnisse werden nun mit den erwähnten Studien von Kubiak (2018) und Flack (2016) verglichen, um Anhaltspunkte für Kontinuität und Wandel in den Konstruktionen ostdeutscher Identität zu identifizieren. Beide jüngeren Studien kommen zu dem Ergebnis, dass die in der DDR oder in Ostdeutschland Geborenen sowohl eine Abwertung der Ostdeutschen in der öffentlichen Debatte wahrnehmen als auch individuell in bestimmten Situationen erfahren. Diese Abwertung bilde den Ausgangspunkt ihrer Identitätskonstruktionen (Kubiak 2018, S. 35; Flack 2016, S. 67) und es finden sich einige - jedoch nicht alle - der bereits für 2004 beschriebenen Reaktionsstrategien wieder. Kubiak (2018, ebd.) berichtet davon, dass die nach 1990 Geborenen eine Ost-Kategorisierung mit Verweis auf die kulturelle Einheit Gesamtdeutschlands ablehnen und delegitimieren, sich aber im Falle einer Fremddefinition dennoch individuell als ostdeutsch identifizieren. Auch ist ebenso wie $2004 \mathrm{zu}$ beobachten, dass die Frage nach der Ost-West-Kategorisierung sowohl von den vor als auch den nach 1990 Geborenen eher als ein Problem der älteren Generation wahrgenommen wird (ebd., S. 35; Flack 2016, S. 67). Die vor 
1990 Geborenen reagierten auf die wahrgenommene Abwertung der Ostdeutschen mit dem Versuch, ein alternatives Bild ostdeutscher Identität in der Öffentlichkeit zu etablieren, um die wahrgenommene Hegemonie des medial vermittelten Identitätsangebots „ostdeutsch“ aufzubrechen und durch differenziertere ostdeutsche Identitäten zu ersetzen (Flack 2016, S. 63). Die von Kubiak (2018) beschriebenen Identitätskonstruktionen spiegeln somit die 2004 identifizierten Typen der Kontext-Ostdeutschen wider. In Flacks (2016) Interviews können hingegen die Integrativen Identifizierer wiedererkannt werden. Diese Zuordnung wird dadurch unterstrichen, dass Flack (2016, S. 64) die 2004 bei den Integrativen Identifizierern konstatierte interne Heterogenität und deren gering ausgeprägte Artikulation von Stereotypisierungen in gleicher Weise bei ihren Interviewpartner/-innen findet. Einige Typen aus dem Jahr 2004 sind deshalb auch noch rund ein Jahrzehnt später zu erkennen, wodurch die partielle Kontinuität in der Konstruktion ostdeutscher Identitäten deutlich wird. Die Validität dieses Befundes wird durch die partiellen Unterschiede in Datenerhebung und Fallauswahl bekräftigt.

Dennoch zeigen sich Hinweise auf diachrone Unterschiede. Da Kubiak und Flack in ihren jeweiligen Interpretationen die Identitätskonstruktion $\mathrm{zu}$ einem einzigen Typus verdichten, ist die 2004 deutlich gewordene interne Heterogenität der ostdeutschen Identitätsbildungsprozesse in den beiden jüngeren Studien nicht mehr in gleicher Weise präsent. Dieser Unterschied könnte auf die variierenden Forschungsdesigns zurückgehen. Bei Kubiak (2018) stellt sich die Frage, ob die Homogenität des sozialen Status der Interviewten als Studierende zu einer größeren Übereinstimmung in den Gruppendiskussionen als 2004 geführt hat. Bei Einbezug anderer Bildungsgruppen und damit einer größeren Heterogenität des sozialen Status - wie in der Studie von 2004 - wäre zu vermuten, dass insbesondere die Typen Abwertungsund Aufwertungsidentifizierer erneut auftauchen würden, deren Identitätsbildung als „ostdeutsch“ eng mit Erfahrungen individueller und kollektiver Deprivation einhergeht. Zudem könnte die Homogenität der Identitätsbildungsprozesse auf der internen Dynamik der Gruppendiskussion beruhen, die - begünstigt durch die soziale Homogenität - zu einer wechselseitigen Anpassung geführt haben könnte.

Für die Einzelinterviews von Flack (2016) gilt die letztgenannte Einschränkung nicht, wohingegen der soziale Status der Interviewten unbekannt ist. Allerdings ist hierbei zu prüfen, ob die thematische Rahmung der Interviews im Kontext der Bemühungen zur politischen Etablierung einer Generation von „Wendekindern“ (s. oben) und die damit zusammenhängende Auswahl der Probanden zur Betonung der Homogenität dieser Generationserfahrung beigetragen hat. Es ist z. B. auffällig, dass die von Flack selbst erwähnte Heterogenität der Identitäten dieser Generation nicht anhand von Interviewpassagen gezeigt wird, sondern dafür auf externe Ereignisse verwiesen wird (ebd., S. 63).

Die methodischen Unterschiede und möglichen Selektionseffekte können also dazu geführt haben, dass in beiden jüngeren Studien jeweils nur ein einziger Typus ostdeutscher Identitätsbildung identifiziert wurde. Dafür spricht, dass der synchrone Vergleich der beiden jüngeren Studien vor dem Hintergrund der Ergebnisse von 2004 zeigt, dass die interne Heterogenität ostdeutscher Identitäten fortbesteht. Weil Flack (2016) unter den zwischen 1975 und 1985 Geborenen nur den Typus der Integrativen Identifizierer fand und Kubiak (2018) unter den nach 1990 Geborenen nur 
die Kontext-Ostdeutschen, zeigt sich, dass diese Heterogenität plausibel auf Kohortenunterschiede zurückgeführt werden kann, wenn die individuelle Erfahrung in der DDR oder deren Fehlen als eine die Kohorten prägende Erfahrung berücksichtigt wird. Vor dem Hintergrund der Ergebnisse von 2004 legt der Vergleich somit nahe, dass die jüngeren Studien nur einen Ausschnitt aus dem Spektrum ostdeutscher Identitätskonstruktionen erfasst haben und dass eine heterogenere Gruppenkomposition der Interviewten - z. B. nach Bildungsgrad, sozialen Status oder Alter - vermutlich weitere Typen ostdeutscher Identität zeigen würde.

Das ist insbesondere relevant, weil die 2004 identifizierten Typen Abgrenzungsund Aufwertungsidentifizierer, die die Ost-West-Kategorisierung betonen, um sich damit von Westdeutschland abzugrenzen und Ostdeutschland aufzuwerten, in den Studien circa ein Jahrzehnt später nicht mehr vorkommen. Auch die Nur-Klassifizierer und Ablehner der Klassifizierung, die die Ost-West-Kategorisierung ablehnen und sich selbst bei Fremddefinition als ostdeutsch nicht mit Ostdeutschland identifizieren, sind ebenso nicht präsent - die in Kubiaks (2018) Studie vertretenen KontextOstdeutschen identifizieren sich trotz Ablehnung der Kategorisierung kontextabhängig doch als Ostdeutsche (ebd., S. 35). Hier stellt sich umso mehr die Frage, ob dieses Ergebnis bei Kubiak (2018) durch die soziale Komposition der Interviewten erklärt werden kann, sind doch Abwertungserfahrungen und die kollektive Gegenreaktion der Aufwertung der Eigengruppe eng mit dem sozialen Status verknüpft. Bei Flack (2016) kann die Rahmung der Interviews und Auswahl des Samples im Kontext eines politischen Netzwerkes zur Etablierung einer ,Wendekindergeneration" dazu geführt haben, dass die eigene Ostidentität bewusst betont wird und sich von kollektiven Strategien, wie bei den vorherigen „Ostgenerationen“ beobachtet, abgegrenzt wird.

Ungeachtet möglicher methodischer Ursachen für einen Teil der Befundlage eröffnen sich damit Anhaltspunkte für Vergleichsperspektiven. Der Interkohortenvergleich der Ergebnisse von 2004 mit Kubiak (2018) eröffnet die Möglichkeit der Untersuchung von Kohorten- oder Generationseffekten. Für die nach 1990 Geborenen bzw. mindestens für diejenigen unter ihnen mit akademischer Prägung und damit geringerer individueller Deprivationserfahrung - spielt die ostdeutsche Identität allein in Situationen der Fremddefinition eine Rolle, wird aber zugleich grundsätzlich abgelehnt. Diese Wahl der individuellen Strategie als Reaktion auf die Fremddefinition als „ostdeutsch“ kann Kennzeichen dafür sein, dass die nach 1990 Geborenen die Gruppengrenze zwischen Ost und West als durchlässiger empfinden als die älteren Kohorten, die eher auf die Umdeutung der relevanten Vergleichsdimension und die Aufwertung der kollektiven Identität setzen. Dass ihre Ablehnung der Kategorisierung aber nicht zu einer generellen Ablehnung der individuellen Identifikation als „ostdeutsch“ führt, ist möglicherweise Ergebnis einer identitätspolitischen Wahrnehmung, in der die Zugehörigkeit zu benachteiligten Gruppen unter bestimmten Umständen der individuellen Mobilität nicht hinderlich, sondern sogar dienlich ist. Dass die jüngere Kohorte ihre ostdeutsche Identität dabei weniger ostentativ betont als die (älteren) Integrativen Identifizierer 2004 und bei Flack (2016) könnte Hinweis sein auf eine eher instrumentelle Identitätsbildung, die durch Geburt und Sozialisation nach 1990 erleichtert wird. 


\section{Fazit}

Der Intra- und Interkohortenvergleich der Ergebnisse von drei qualitativen Studien zur Konstruktion ostdeutscher Identitäten unterstreicht, dass eine vergleichende Analyse des zugrundeliegenden Datenmaterials und ihre Erweiterung um sozial heterogenere Interviewsamples eine Bereicherung der Diskussion um ostdeutsche Identitäten mit sich bringen kann. Der Vergleich verdeutlicht erstens, dass eine partielle Kontinuität in den Identitätsbildungsprozessen zwischen 2004 und einer Dekade später besteht. Stellen kollektive Identitäten eine Symbolisierung von Gruppenverhältnissen dar, besteht die wahrgenommene Asymmetrie zwischen Ost- und Westdeutschland in Teilen offenbar fort. Zweitens artikulieren die Interviewten der drei Studien trotz eines von ihnen als dominant wahrgenommenen Identitätsangebots nicht eine einheitliche ostdeutsche Identität, sondern eine Vielzahl ostdeutscher Identitäten, die mit unterschiedlichen Folgen für ihre Handlungsorientierungen einhergehen. „Ostdeutsch“ wird weiterhin oft als abwertende Klassifizierung wahrgenommen, auf die mit individueller Mobilität und Praktiken der De- und Reklassifizierung reagiert wird - sowohl im Erhebungsjahr 2004 als auch rund eine Dekade später. Ostdeutsche Identitäten entstehen jedoch nicht ausschließlich als Reaktion auf wahrgenommene Abwertung, sondern sind auch Ergebnis der Erfahrungen der Transformation in Ostdeutschland. In diesen Fällen wird die erfolgreiche Bewältigung dieser Transformation zur Basis der ostdeutschen Identität gemacht, die nicht abgrenzend ist, sondern als Bereicherung gesamtdeutscher Diskussions- und Entscheidungsprozesse verstanden wird. Schließlich konnten einige Typen ostdeutscher Identität, die 2004 noch präsent waren, nicht erneut gezeigt werden, insbesondere solche, bei denen Abwertungserfahrungen durch die Strategie der Umdeutung der Vergleichsdimension und der kollektiven Aufwertung der Gruppe der Ostdeutschen kompensiert werden. Aufgrund der geschilderten methodischen Vorbehalte können jedoch erst weitere Analysen zeigen, ob diese Typen nicht mehr in der Bevölkerung präsent sind und ob daher individuelle und instrumentelle Identitätsbildungsprozesse gegenwärtig überwiegen. Für diese Analyse böte sich eine Reproduktion früherer Befragungen unter Konstanthaltung der Struktur der Interviewsamples sowie des Erhebungsinstruments und seiner inhaltlichen Kontextualisierung an. Drittens lässt sich die geschilderte Heterogenität plausibel auf biografische Unterschiede und Kohorteneffekte zurückführen. Zukünftige Studien sollten deshalb verstärkt die sozialen und politischen Eigenschaften der Befragten sowie die Randbedingungen für deren Identitätskonstruktionen in den Blick nehmen.

Funding Open Access funding enabled and organized by Projekt DEAL.

Open Access Dieser Artikel wird unter der Creative Commons Namensnennung 4.0 International Lizenz veröffentlicht, welche die Nutzung, Vervielfältigung, Bearbeitung, Verbreitung und Wiedergabe in jeglichem Medium und Format erlaubt, sofern Sie den/die ursprünglichen Autor(en) und die Quelle ordnungsgemäß nennen, einen Link zur Creative Commons Lizenz beifügen und angeben, ob Änderungen vorgenommen wurden.

Die in diesem Artikel enthaltenen Bilder und sonstiges Drittmaterial unterliegen ebenfalls der genannten Creative Commons Lizenz, sofern sich aus der Abbildungslegende nichts anderes ergibt. Sofern das betreffende Material nicht unter der genannten Creative Commons Lizenz steht und die betreffende Handlung 
nicht nach gesetzlichen Vorschriften erlaubt ist, ist für die oben aufgeführten Weiterverwendungen des Materials die Einwilligung des jeweiligen Rechteinhabers einzuholen.

Weitere Details zur Lizenz entnehmen Sie bitte der Lizenzinformation auf http://creativecommons.org/ licenses/by/4.0/deed.de.

Interessenkonflikt L. Vogel und J. Leser geben an, dass kein Interessenkonflikt besteht.

\section{Literatur}

Ahbe, Thomas. 2004. Die Konstruktion der Ostdeutschen. Diskursive Spannungen, Stereotype und Identitäten seit 1989. Aus Politik und Zeitgeschichte B41-42:12-22.

Best, Heinrich, Axel Salheiser, und Katja Salomo. 2014. Demokratie mit doppelter Diktaturvergangenheit. Überlegungen und Befunde zur Ausprägung eines spezifisch ostdeutschen Rechtsextremismus. In Kein Ende mit der Wende? Perspektiven aus Ost und West, Hrsg. Elmar Brähler, Wolf Wagner, 149-167. Gießen: Psychoszial-Verlag.

Brunner, Wolfram, und Dieter Walz. 1998. Warum sich die Ostdeutschen zwar als „Bürger zweiter Klasse“ fühlen, wir aber nicht auf die innere Mauer treffen. In Werte und nationale Identität im vereinten Deutschland. Erklärungsansätze der Umfrageforschung, Hrsg. Heiner Meulemann, 229-250. Opladen: Leske + Budrich.

Delitz, Heike. 2018. Kollektive Identitäten. Bielefeld: transcript.

Emcke, Carolin. 2018. Kollektive Identitäten: Sozialphilosophische Grundlagen. Frankfurt a. M.: S. Fischer.

Fach, Wolfgang, Karl-Christian Köhnke, Matthias Middell, Kurt Mühler, Hannes Siegrist, Sabine Tzschaschel, und Heinz-Werner Wollersheim. 1998. Regionenbezogene Identifikationsprozesse. Das Beispiel „Sachsen“ - Konturen eines Forschungsprogramms. In Region und Identifikation, Hrsg. HeinzWerner Wollersheim, Sabine Tzschaschel, und Matthias Middell, 1-32. Leipzig: Leipziger Universitätsverlag.

Flack, Jaqueline. 2016. Zwischen Anrufung und Subjektivierung: Diskursive und narrative Praxen ostdeutscher Identitätskonstruktionen nach 1989 am Beispiel der Wendegeneration. In Die Generation der Wendekinder: Elaboration eines Forschungsfeldes, Hrsg. Adriane Lettrari, Christian Nestler, und Nadja Troi-Boeck, 55-69. Wiesbaden: Springer VS.

Foroutan, Naika, Frank Kalter, Coşkun Canan, und Mara Simon. 2019. Ost-Migrantische Analogien I. Konkurrenz um Anerkennung. Berlin: DeZIM-Institut. Unter Mitarbeit von Daniel Kubiak und Sabrina Zajak.

Hacker, Michael, Stephanie Maiwald, Johannes Staemmler, Judith Enders, Adriana Lettrari, Hagen Pietzcker, Henrik Schober, und Mandy Schulze (Hrsg.). 2012. Dritte Generation Ost: Wer wir sind, was wir wollen. Berlin: Links.

Hacking, Ian. 1999. The social construction of what? Cambridge: Harvard University Press.

Haeger, Gabriele. 1998. Wächst wirklich zusammen, was zusammengehört? Identität und Wahrnehmung der Intergruppensituation in Ost- und Westdeutschland. Münster: LIT.

den Hertog, Frank. 2004. Minderheit im eigenen Land? zur gesellschaftlichen Position der Ostdeutschen in der gesamtdeutschen Realität. Frankfurt am Main, New York: Campus.

Jenkins, Richard. 1996. Social identity. London, New York: Psychology Press.

Kaase, Max, und Petra Bauer-Kaase. 1998. Deutsche Vereinigung und innere Einheit 1990-1997. In Werte und nationale Identität im vereinten Deutschland. Erklärungsansätze der Umfrageforschung, Hrsg. Heiner Meulemann, 251-268. Opladen: Leske + Budrich.

Kollmorgen, Raj. 2011. Subalternisierung: Formen und Mechanismen der Missachtung Ostdeutscher nach der Vereinigung. In Diskurse der deutschen Einheit: Kritik und Alternativen, Hrsg. Raj Kollmorgen, Frank Thomas Koch, und Hans-Liudger Dienel, 301-359. Wiesbaden: VS.

Kollmorgen, Raj, und Torsten Hans. 2011. Der verlorene Osten. Massenmediale Diskurse über Ostdeutschland und die deutsche Einheit. In Diskurse der deutschen Einheit: Kritik und Alternativen, Hrsg. Raj Kollmorgen, Frank Thomas Koch, und Hans-Liudger Dienel, 107-166. Wiesbaden: VS.

Kubiak, Daniel. 2018. Der Fall „Ostdeutschland“: „Einheitsfiktion“ als Herausforderung für die Integration am Fallbeispiel der Ost-West-Differenz. Zeitschrift für Vergleichende Politikwissenschaft 12:25-42.

Lettrari, Adriana, Christian Nestler, und Nadja Troi-Boeck (Hrsg.). 2016. Die Generation der Wendekinder: Elaboration eines Forschungsfeldes. Wiesbaden: Springer VS.

Mayring, Philipp. 2015. Qualitative Inhaltsanalyse: Grundlagen und Techniken, 12. Aufl., Weinheim Basel: Beltz. 
Mummendey, Amélie, und Thomas Kessler. 2000. Deutsch-deutsche Fusion und soziale Identität: Sozialpsychologische Perspektiven auf das Verhältnis von Ost- zu Westdeutschen. In Der Wandel nach der Wende, Hrsg. Hartmut Esser, 277-307. Wiesbaden: Westdeutscher Verlag.

Pates, Rebecca. 2013. Einleitung - Der „Ossi“ als symbolischer Ausländer. In Der „Ossi“: Mikropolitische Studien über einen symbolischen Ausländer, Hrsg. Rebecca Pates, Maximilian Schochow, 7-20. Wiesbaden: Springer VS.

Pickel, Gert, und Detlef Pollack. 1998. Die ostdeutsche Identität - Erbe des DDR-Sozialismus oder Produkt der Wiedervereinigung? Aus Politik und Zeitgeschichte B41-42(98):9-23.

Quent, Matthias. 2012. Rechtsextremismus - ein ostdeutsches Phänomen? Aus Politik und Zeitgeschichte 62(16/17):38-42.

Reiser, Marion, Heinrich Best, Axel Salheiser, und Lars Vogel. 2018. Heimat Thüringen: Ergebnisse des THÜRINGEN-MONITORS 2018. Drucksache des Thüringer Landtags 6/6383. https://www. landesregierung-thueringen.de/regierung/th-monitor/.

Rippl, Susanne, Nelly Buntfuß, Nicole Malke, Natalie Rödel, und Luisa Schubert. 2018. Ostdeutsche Identität: Zwischen medialen Narrativen und eigenem Erleben. Deutschland Archiv 16.5.2018. www.bpb. de/269349. Zugegriffen: 30. Mai 2020.

Roth, Kersten Sven. 2008. Der Westen als „Normal-Null“. Zur Diskurssemantik von „ostdeutsch“ und „westdeutsch“. In Diskursmauern. Aktuelle Aspekte der sprachlichen Verhältnisse zwischen Ost und West, Hrsg. Kersten Sven Roth, Markus Wienen, 69-89. Bremen: Hempen.

Scherr, Albert. 1995. Soziale Identitäten Jugendlicher. Politische und berufsbiographische Orientierungen von Auszubildenden und Studenten. Wiesbaden: Springer VS.

Tajfel, Henri, und John C. Turner. 2004. The social identity theory of intergroup behaviour. In Key readings in social psychology. Political psychology: Key readings, Hrsg. John T. Jost, Jim Sidanius, 276-293. London: Psychology Press.

Wagner, Peter. 1999. Fest-Stellungen. Beobachtungen zur sozialwissenschaftlichen Diskussion über Identität. In Identitäten. Erinnerung, Geschichte, Identität, Hrsg. Aleida Assmann, Heidrun Friese, 44-72. Frankfurt a. M.: Suhrkamp. 